\title{
USO DEL ARCO EXTRAORAL EN LA CORRECCIÓN DE LA MALOCLUSIÓN CLASE II, DIVISIÓN 2, TIPO C
}

\section{USE OF THE EXTRAORAL ARCH IN THE CORRECTION OF CLASS II, DIVISION 2, TYPE C MALOCCLUSION}

\author{
Juan Carlos Loza Campos ${ }^{1 a}$, Juan Carlos Chancafe Morgan²ab ${ }^{2}$ Ana Cecilia Lavado Torres ${ }^{1 \mathrm{abc}}$.
}

\section{RESUMEN}

Se presenta el caso de una paciente de 13 años de edad, a quien se le realizó el tratamiento de una maloclusión clase II, división 2, tipo C, mediante el uso del arco extraoral teniendo en cuenta la edad del paciente y la etapa de maduración esquelética en la que se encontraba. En este artículo presentamos el uso de las fuerzas extraorales y los cambios esqueletales, faciales y dentarios que finalmente se consiguieron.

Palabras Clave: ortodoncia, aparatos de tracción extraoral, Maloclusión de Angle Clase II . (Fuente: DeCS BIREME)

\section{ABSTRACT}

This case report presents a 13 year old patient who underwent a class II division 2 type $\mathrm{C}$ malocclusion treatment with an extraoral headgear, regarding patient's age and skeletal maduration stage at that moment. This article presents the use of extraoral forces and the skeletal, facial and dental changes that were finally achieved.

Keywords: orthodontics, Extraoral Traction Appliances, Malocclusion, Angle Class II . (Source: MeSH NLM)

${ }^{1}$ Universidad San Martin de Porres

${ }^{2}$ Universidad Peruana Cayetano Heredia

${ }^{a}$ Especialista en Ortodoncia y Ortopedia maxilar

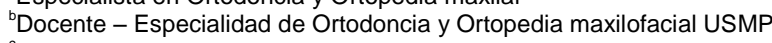

'Doctora en Educación

\section{Correspondencia:}

Juan Carlos Loza Campos

Teléfono: 0051997499353

Correo electrónico: juancarlosloza_c@hotmail.com
Este es un artículo Open Access distribuido bajo la licencia Creative Commons Atribución-NoComercial- Compartir Igual 4.0

\section{(๑)@(○)}

Citar como: Loza - Campos JC, Chancafe - Morgan JC, Lavado - Torres AC. Uso del arco extraoral en la corrección de la maloclusión clase II, división 2, tipo C. KIRU. 2018; 15(1): 42 - 47. https://doi.org/10.24265/kiru.2018.v15n1.05 


\section{INTRODUCCIÓN}

La maloclusión se define como cualquier alteración del crecimiento óseo del maxilar o mandíbula y/o de las posiciones dentarias que impidan una correcta función del aparato masticatorio. ${ }^{(1)}$

Vellini, nombró al Dr. Angle en el que se proponía clasificar las maloclusiones y propuso dividirlas en clases: I, II y III. ${ }^{(2)}$

Una maloclusión de clase II se define como la posicion mesial de la primera molar superior con respecto a la primera molar inferior. Se presenta como una incorrecta relación entre los arcos maxilares y mandibulares debido a problemas esqueléticos, problemas dentales o una combinación de ambos. ${ }^{(3)}$

El beneficio de conocer la etapa de madurez esqueletal permitirá adoptar medidas respecto a las terapias ortopédicas que se indiquen en pacientes con anomalías esqueletales. Por ello, la ventaja de aprovechar el periodo de crecimiento más acelerado permite estimular aún más la respuesta biológica del desarrollo. ${ }^{(4,5)}$

\section{REPORTE DE CASO CLÍNICO}

Paciente de 13 años de edad, de sexo femenino, simétrico, mesofacial, hiperdivergente con un perfil convexo. Presenta una sonrisa simétrica con una musculatura hipertónica.

Dentro del examen intraoral presenta dentición permanente, relaciones molares derecha de clase II (75\%) e izquierda de clase II $(50 \%)$, relación canina derecha e izquierda clase II (100\%), línea media dentaria superior centrada con respecto a la línea media facial y la línea media dentaria inferior desviada $1 \mathrm{~mm}$ a la derecha con respecto a la línea media dentaria superior, overjet de $+4 \mathrm{~mm}$, overbite de $5 \mathrm{~mm},(62,5 \%)$, curva de Spee: $1,5 \mathrm{~mm}$.
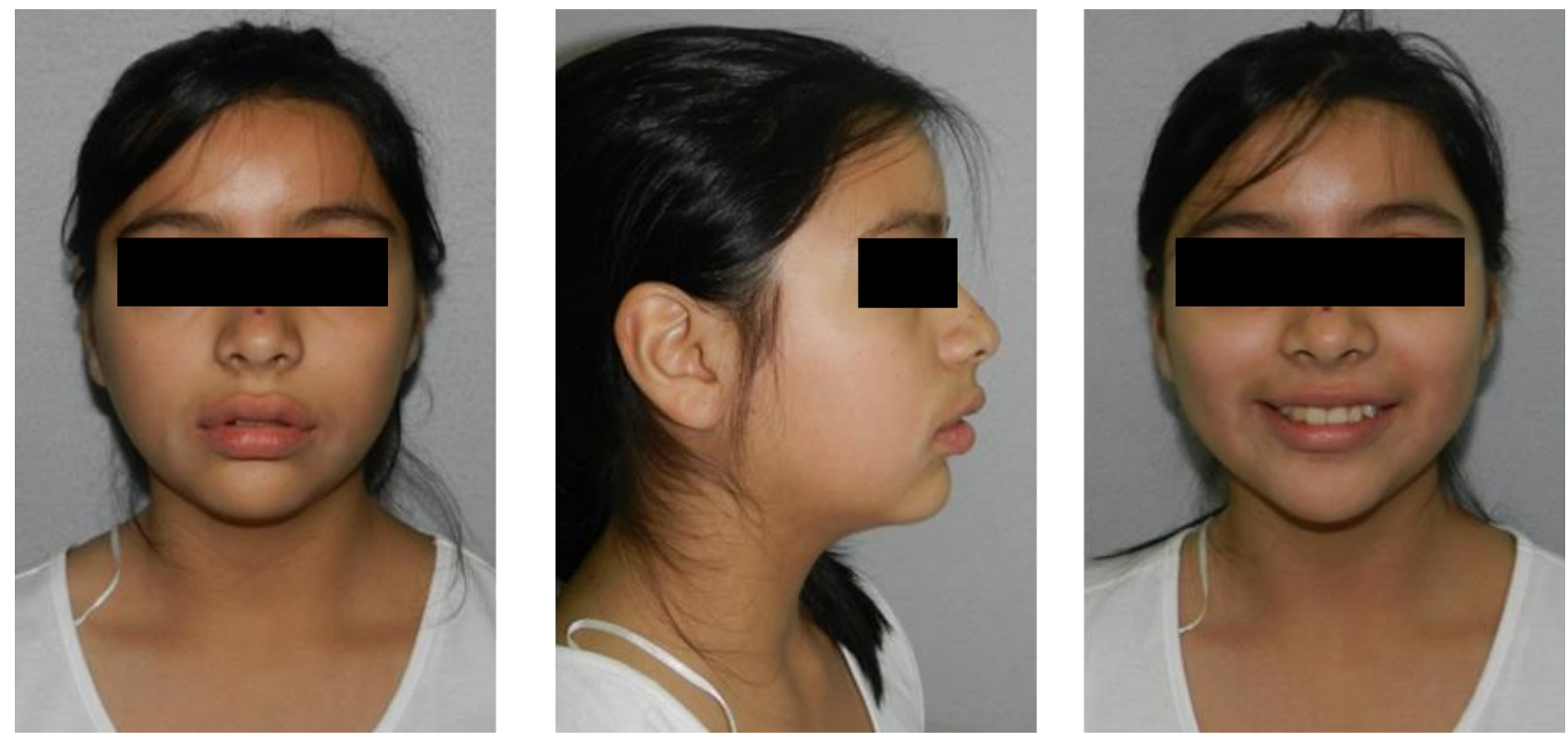

Figura 1. Fotografías extraorales iniciales. 

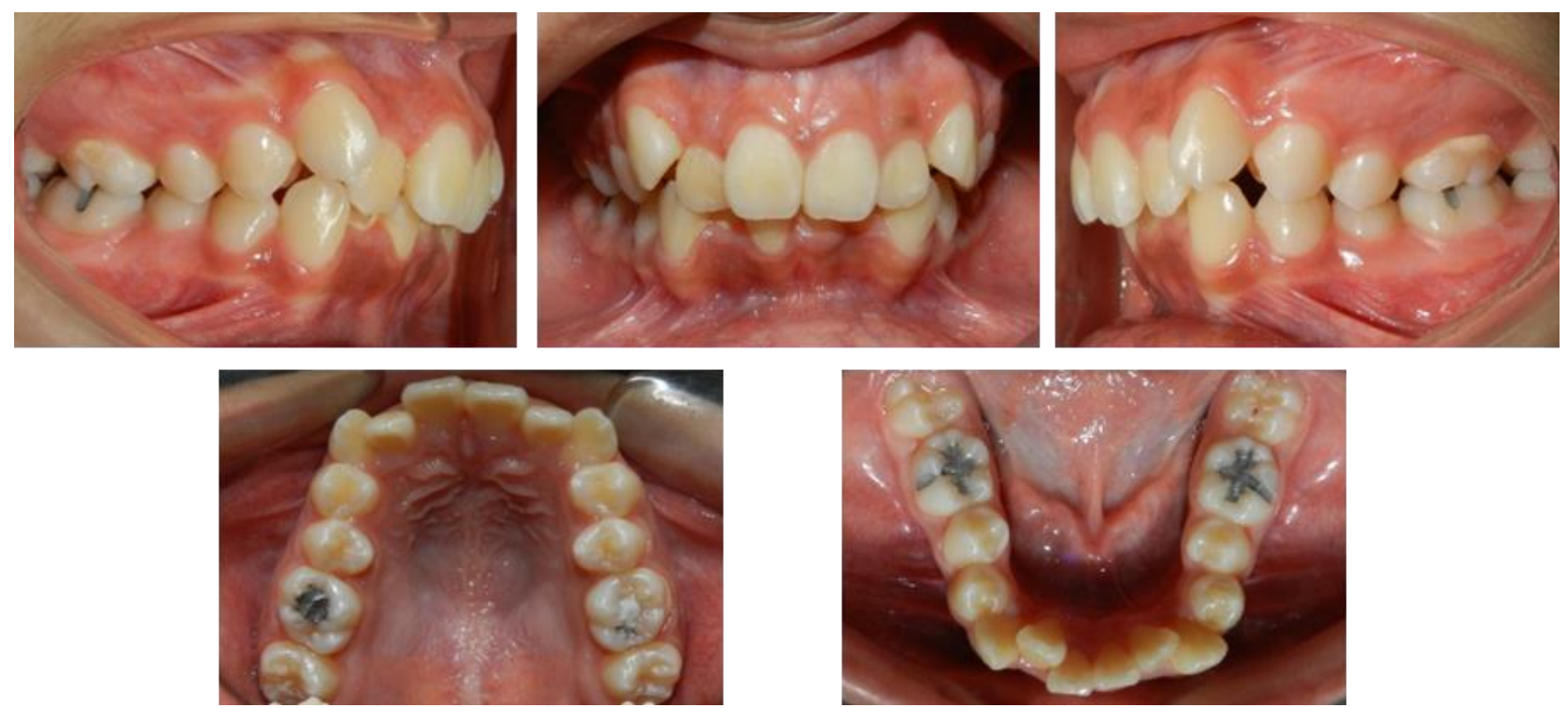

Figura 2. Fotografías intraorales iniciales.

Se inicia el tratamiento con la fase de alineamiento y nivelación para el maxilar superior con un arco transpalatino, soldado a bandas con tubos triples convertibles ubicados en las primeras molares permanentes superiores. Se indicaron extracciones de los primeros premolares superiores e inferiores.
Se trabajó simultáneamente con un arco extraoral (AEO) de tracción media alta. Se indicó su uso durante 14 horas diarias por un periodo de un año y medio, como también el empleo de elásticos de $1 / 2$ pulgada (12 onzas), ejerciendo una fuerza ortopédica de 450 gramos por lado con el fin de redireccionar el crecimiento del maxilar superior.
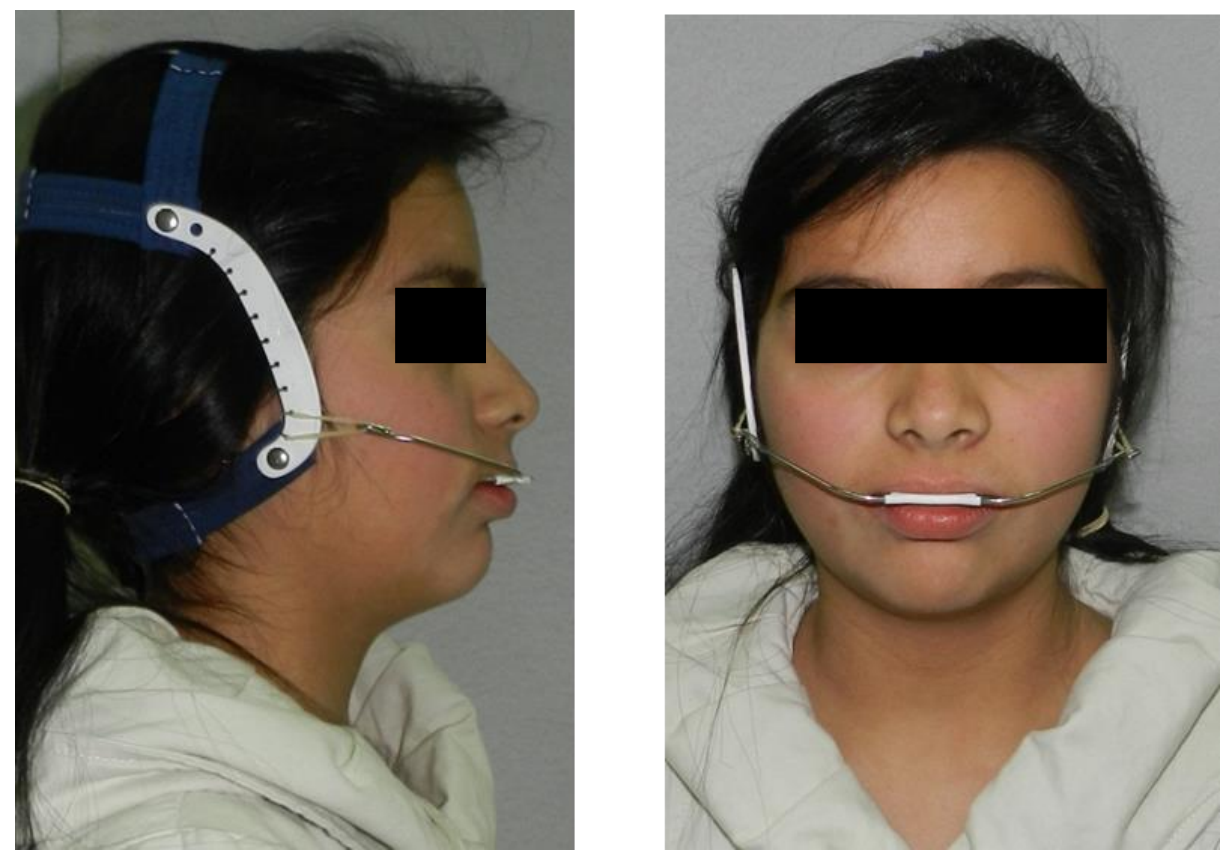

Figura 3. AEO tracción media alta. 
Finalizada la primera fase, se inicia el cierre de espacios, realizándolo en dos tiempos: primero la distalización de los caninos y luego el sector anterior para ambas arcadas. El cierre de espacios de los cuatro dientes anteriores se hizo con la mecánica por desplazamiento o sin fricción por medio de ansas de cierre con un arco de acero 0,017 x 0,025", ayudado de curvas de compensación para el control vertical y evitar un colapso de mordida. De esta manera se logró conseguir correctas relaciones oclusales.
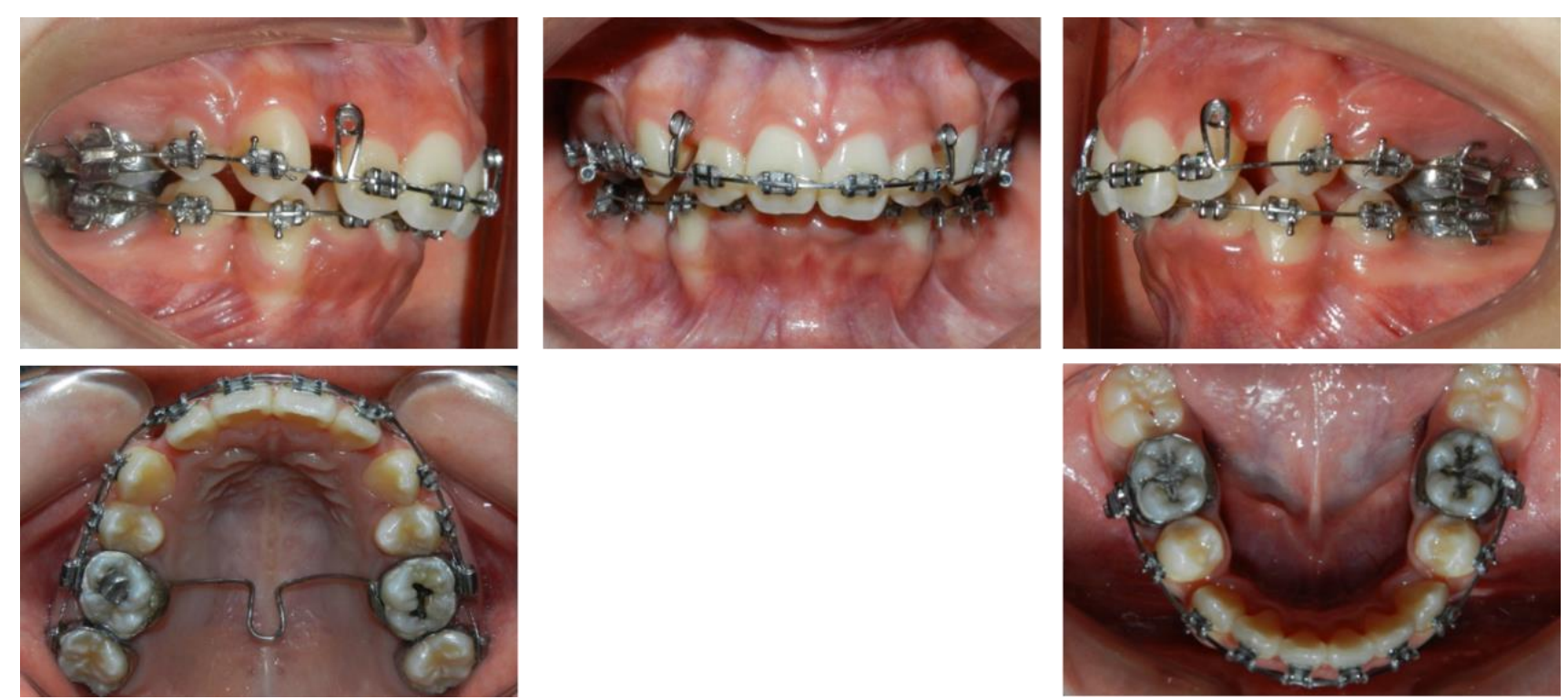

Figura 4. Fotos intraorales de progreso, etapa de cierre de espacios con ansas de cierre

Para finalizar, en la etapa de acabado e intercuspidación se tomó como referencia la radiografía panorámica y se reposicionaron algunos brackets con el fin de conseguir un correcto engranaje oclusal. Se utilizaron arcos de acero de $0,017 \times 0,025$ " en el maxilar superior e inferior, con los cuales se procedió a realizar dobleces de primer, segundo y tercer orden; posteriormente, se indicó el uso de elásticos intermaxilares de $3 / 16$ " - 4 onzas de forma triangular bilateral.

\section{RESULTADO DEL TRATAMIENTO}

Los resultados fueron muy buenos, ya que la paciente fue colaboradora en el uso del arco extraoral (AEO) y en la asistencia a sus controles mensuales. Se pudo obtener una mejora en la armonía facial y una corrección en las relaciones oclusales. También se consiguió centrar las líneas medias dentarias con respecto a la línea media facial y se lograron los objetivos trazados desde un inicio. 

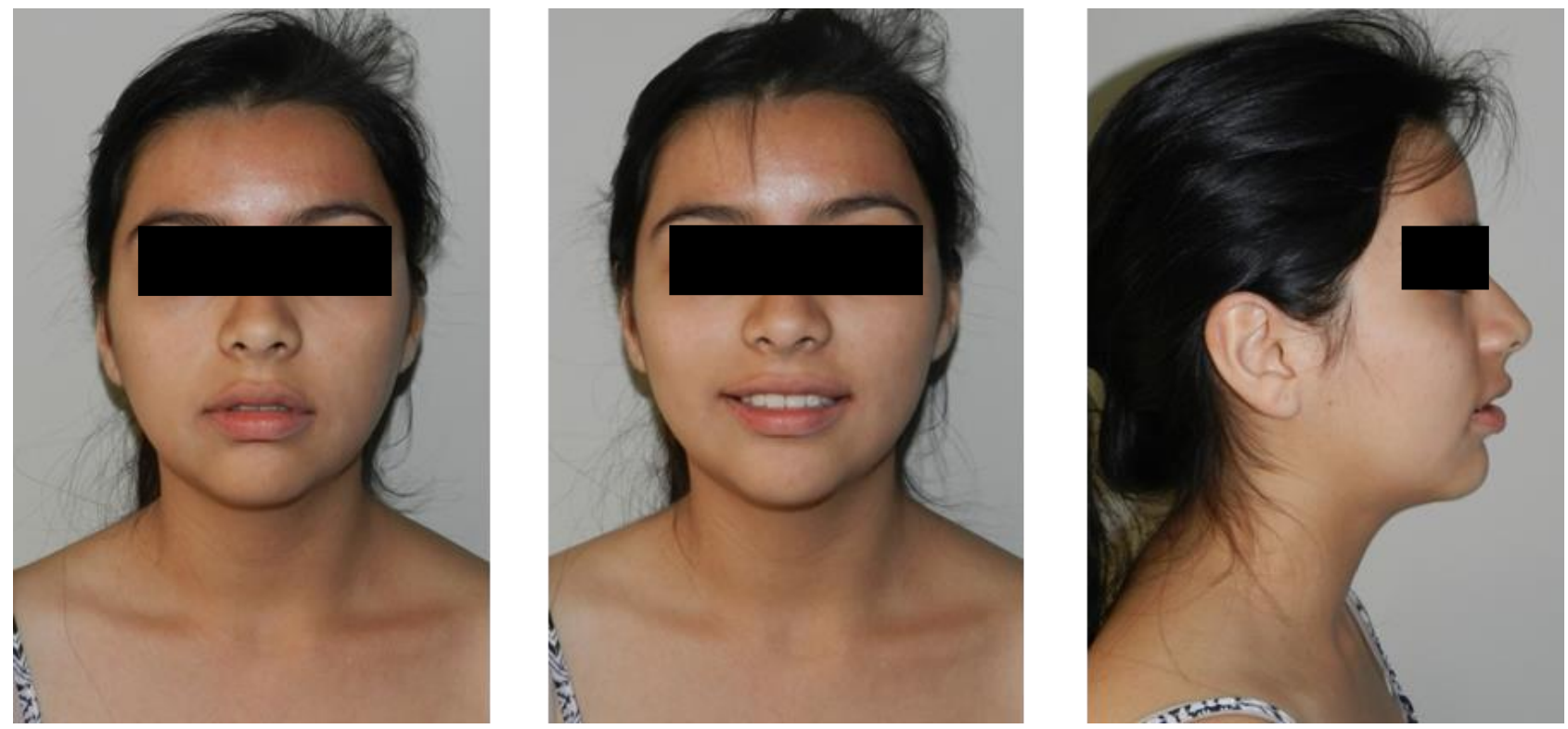

Figura 5. Fotografías extraorales postratamiento. Se puede observar una sonrisa mucho más agradable y una mejora notable en el perfil.por desplazamiento o sin fricción.
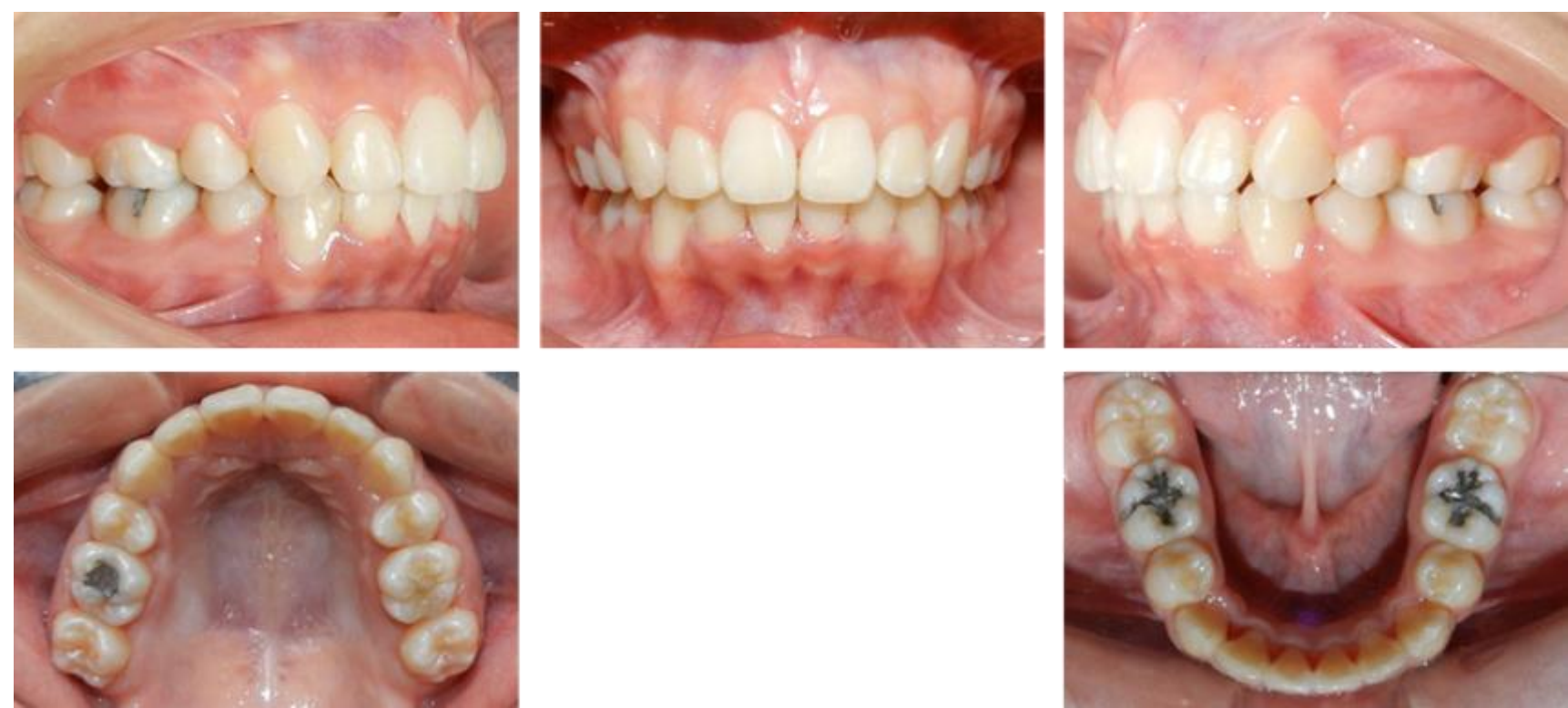

Figura 6. Fotografías intraorales postratamiento. Relaciones molares y caninas de clase I bilateral, líneas medias dentarias centradas con respecto a la línea media facial. 


\section{DISCUSIÓN}

El tratamiento con arco extraoral influye en el crecimiento esquelético maxilofacial. Por ello, esto aplica tanto a la dirección del crecimiento como a la cantidad de crecimiento durante el periodo de tratamiento. No obstante, aún no se ha demostrado que la terapia con ortopedia facial que termina antes del final del crecimiento facial altere el tamaño y la forma de las estructuras esqueléticas a largo plazo. Por otro lado, la relación maxilomandibular puede mejorarse, lo que facilita la corrección de la distooclusión. Además, la mejora no se pierde a largo plazo si se consigue una intercuspidación sólida de los premolares al final del tratamiento. ${ }^{(3)}$

En los tratamientos ortopédicos con fuerzas extraorales se debe tener en cuenta la dirección del crecimiento cráneo facial, la sensibilidad vertical, la altura facial antero inferior y la sobremordida vertical dental del paciente, ya que, dependiendo de la magnitud, la dirección y la duración de la fuerza, se afectará la relación maxilomandibular. ${ }^{(4)}$

En el presente caso clínico, se consiguió una reducción del patrón esquelético por redirección del crecimiento, debido al uso de las fuerzas extraorales, procedimiento que ha mostrado ser eficaz ${ }^{(6)}$. Los resultados favorables que se consiguieron han dependido mucho de la colaboración de la paciente y el uso del arco extraoral 16 horas diarias en promedio. $^{(7)}$

Es importante evaluar de forma adecuada e identificar posibles alteraciones oportunamente, así como realizar una selección adecuada del tratamiento porque esto permitirá aplicar un tratamiento interceptivo que pueda eliminar o reducir la severidad de una y se maloclusión se reflejará en la satisfacción, del paciente y en el cumplimiento de los objetivos terapéuticos ${ }^{(8,9)}$.

\section{CONCLUSIONES}

1. El uso del arco extraoral corrigió las posiciones de las molares y mejoró la de los incisivos. La colaboración del paciente y el uso diario del arco extraoral fueron factores importantes para el éxito de la terapia; por ello, hay que tener en cuenta los principios biomecánicos para el correcto uso de la aparatología, lo que nos llevará a una terapia eficiente y en un menor plazo.

2. Es importante tener en consideración la etapa de maduración esqueletal en que se encuentra el paciente, lo que va ser clave para obtener un óptimo resultado en un tratamiento ortopédico.

3. La literatura aporta muchos conceptos y protocolos básicos para el tratamiento con extracciones. Será importante aplicarlos clínicamente sobre la base de la evidencia científica para lograr resultados óptimos en el tratamiento ortodóntico.

\section{REFERENCIAS BIBLIOGRÁFICAS}

1. Interlandi S. Ortodoncia Bases para la iniciación.161-2. Madrid: Editorial Artes Medicas Ltda; 2002.

2. Vellini F. Ortodoncia, Diagnóstico y Planificación Clínica. $2^{a}$. ed. Sao Paulo: Artes Medicas Ltda; 2008.

3. Bishara S. Textbook of orthodontic. Philadephia: W.B. Saunders Company; 2001.

4. Fishman L. Radiographic Evaluation of skeletal maduration. A clinically oriented method bases on hand-wrist film. Angle Orthod.1982; 52(2): 88112.

5. Baccetti T, Franchi L, McNamara J. The cervical vertebral maturation (CVM) method for the assessment of optimal treatment timing in dentofacial orthopedic. Semin Orthod. 2005;11: 119-129.

6. Saldarriaga-Valencia Jenny Angélica, Alvarez Varela Emery, Botero-Mariaca Paola Maria. Tratamientos para la maloclusión Clase II esquelética combinada. CES odontol. [Internet]. 2013 July [cited 2018 May 28] ; 26( 2 ): 145-

159. Available from: http://www.scielo.org.co/scielo.php?script=sci_ar ttext\&pid=S0120-971X2013000200013\&Ing=en.

7. Uribe G. Ortodoncia, teoría y clínica. 2ª ed. Medellín: Ed. Corporación para Investigaciones Biológicas; 2010.

8. Matthews Francisca, Cartes-Velásquez Ricardo. Factores que influyen las decisiones terapéuticas en Ortodoncia: Revisión de la literatura. Odontoestomatología [Internet]. 2017 Jun [citado 2018 Mayo 28] ; 19( 29 ): 33-45. Disponible en: http://www.scielo.edu.uy/scielo.php?script=sci_a rttext\&pid=S1688-93392017000100033\&lng=es.

9. Daza-Aliatis Juan. Prevalencia de maloclusiones en estudiantes universitarios de la Facultad de Odontología. Dom.Cien. 2016; 2: 57-65. Disponible

en: file:///C:/Users/profesor.FOUSMP/Downloads/Di alnet-

PrevalenciaDeMaloclusionesEnEstudiantesUniv ersitar-6325812\%20(1).pdf 